\title{
ESTÁNDARES Y REGULACIÓN DE CALIDAD DE LA FORMACIÓN DE PROFESORES: DISCUSIÓN DEL CASO CHILENO DESDE UNA PERSPECTIVA COMPARADA $^{*}$
}

\section{Carmen Sotomayor, Jacqueline Gysling ${ }^{1}$}

\section{RESUMEN}

El artículo analiza cuatro sistemas de regulación de la calidad de la formación de profesores basados en estándares y discute las medidas que se están desarrollando en Chile en este ámbito. Los casos abordados son Victoria, en Australia; Quebec, en Canadá; Nueva York, en Estados Unidos, e Inglaterra en el Reino Unido, que tienen agencias estatales especializadas y políticas explícitas orientadas a regular la calidad de la formación docente, provista por instituciones universitarias públicas y privadas autónomas. A partir del estudio se observa que, a pesar de las medidas adoptadas, el sistema en Chile se mantendría como el más desregulado en relación con los casos analizados

Palabras clave: formación de profesores, estándares, regulación de la calidad de la formación de profesores, acreditación, certificación de profesores.

\section{QUALITY REGULATIONS AND STANDARDS IN TEACHERS' EDUCATION: THE CHILEAN CASE FROM A COMPARATIVE POINT OF VIEW}

\section{ABSTRACT}

This article analyzes four regulation systems regarding to the quality of teacher education based on standards, and discusses the actions that are being developed on this field in Chile. Cases addressed were: Victoria-Australia, Quebec-Canada, New York-United States, and EnglandUnited Kingdom. All the analyzed cases have specialized government agencies, and an explicit policy oriented to regulate the quality of teacher education provided by public and private universities. Results showed that despite the actions taken, the Chilean system remains as the less regulated when compared to the other analyzed cases.

Keywords: teacher education, standards, quality control in teacher education, accreditation, teacher certification.

\footnotetext{
* Proyecto que contó con el apoyo financiero del Consejo Nacional de Educación (CNED).

1 Centro de Investigación Avanzada en Educación, Universidad de Chile, Santiago, Chile. Contacto: csotoma@dim.uchile.cl
} 


\section{ESTÁNDARES Y REGULACIÓN DE CALIDAD DE LA FORMACIÓN DE PROFESORES: DISCUSIÓN DEL CASO CHILENO DESDE UNA PERSPECTIVA COMPARADA}

\section{Introducción}

El artículo analiza cuatro sistemas de regulación de calidad de la formación de profesores basados en estándares y, a partir de las experiencias descritas, discute las medidas que se están desarrollando en Chile para regular la calidad de la formación docente, destacando aquellos aspectos que pudieran considerarse mayormente en la discusión nacional. Los casos analizados son: el estado de Victoria, en Australia; la provincia de Quebec, en Canadá; el estado de Nueva York, en Estados Unidos, e Inglaterra en el Reino Unido. En estos casos, existen agencias estatales especializadas y una política explícita del estado orientada a regular la calidad de la formación docente, en el marco de un sistema de provisión mixto, donde distintas instituciones públicas y privadas realizan la preparación de los futuros profesores. Los estándares son los referentes que ordenan la política de regulación, están centrados principalmente en desempeños prácticos y son generales a los distintos tipos de profesores. Los casos analizados presentan diferencias en la forma en que articulan los estándares con los procesos de acreditación institucional, que buscan monitorear la calidad de los programas de formación, y con los procesos de certificación, que buscan monitorear las capacidades individuales de los nuevos profesores. No obstante, en todos ellos la acreditación de los programas de formación es obligatoria y existe un registro centralizado de los nuevos profesores. El estudio realizado muestra que las medidas que se están adoptando en Chile en este ámbito no aseguran que se constituya un sistema articulado de regulación basado en estándares que considere la acreditación de las carreras de pedagogía, la certificación para ejercer la profesión y el registro de los nuevos profesores. Dado que la acreditación de las carreras no es totalmente obligatoria, que la prueba Inicia será un sistema 
voluntario de información y que no se prevé un registro centralizado de los nuevos docentes, el sistema en Chile se mantendrá como el más desregulado en relación con los casos analizados.

\section{Antecedentes}

\subsection{Estándares y regulación de la formación de profesores}

En las últimas décadas los estándares han sido pieza clave en la regulación de los sistemas educacionales. Estos han surgido como la respuesta operativa para contar con criterios de calidad comunes y una definición compartida de los resultados de aprendizaje que se esperan de los procesos educativos. En educación se han venido desarrollando estándares desde la década del 80, ya sean impulsados por asociaciones de agencias educacionales (Estados Unidos), por los estados nacionales (Nueva Zelanda o Australia) o por acuerdos internacionales (el acuerdo de Bolonia en Europa).

El término estándar define tanto un criterio fijo respecto del cual se juzga el resultado o puede ser también el logro obtenido. Esta definición puede ser de excelencia o puede ser básica. Puede tener una forma cuantitativa como producto de un proceso de puntos de corte (por ejemplo, un determinado puntaje en una escala de evaluación) o puede ser cualitativa, descriptiva o indicativa de determinados ámbitos. Los estándares pueden tener como referencia los inputs del proceso (estándares de contenido), los outputs del proceso (estándares de desempeño) o los procesos mismos (estándares de oportunidades) (Vlasceanu y otros, 2007).

Los estándares de aprendizaje relacionados con el diseño curricular o inputs del proceso, son denominados "estándares de contenido" y definen lo que los estudiantes deben saber, valorar y poder hacer como meta de su proceso educativo. Los estándares relacionados con las evaluaciones nacionales o estaduales que miden los resultados del proceso, son denominados "estándares de desempeño" y describen lo que efectivamente saben y pueden hacer los estudiantes (Ravitch, 1996, Hansche, 1998, Linn y Herman, 2001, Forster, 2002). Se considera que ambos tipos de estándares deben ser 
94 ESTÁNDARES Y REGULACIÓN DE CALIDAD DE LA FORMACIÓN DE PROFESORES: DISCUSIÓN DEL CASO CHILENO DESDE UNA PERSPECTIVA COMPARADA - Carmen Sotomayor, Jacqueline Gysling

parte de un sistema que debe estar alineado (Hansche y otros, 1998). El "alineamiento", que no es un concepto preciso y existe debate en la literatura al respecto ${ }^{2}$, consiste básicamente en que los estándares de contenido definen las metas del currículo (del proceso de formación para el cual se están definiendo estándares), ya sea de años, ciclos o terminales, y los estándares de desempeño describen niveles de logro de consecución de estándares de contenido en la evaluación, ya sea nacional, local o de aula.

Es importante destacar que tener estándares no es lo mismo que tener una política basada en ellos (Ferrer, 2006). Una política educativa basada en estándares es una agenda explícita de acciones concertadas para lograr mejoramientos significativos en la calidad educativa. Dado que los estándares pretenden movilizar el sistema hacia un mejoramiento, se debe contemplar como aspecto principal en su definición que estos sean expresión de un amplio acuerdo político que incluya la definición de acciones para conseguir su logro. Esto es clave, ya que compromete la sostenibilidad en el tiempo de los estándares y de la política basada en ellos.

El conocimiento acumulado internacionalmente, luego de tres décadas de políticas basadas en estándares, muestra que no solo es clave el consenso inicial, sino las características de los estándares que se definan y de los mecanismos de aseguramiento que se establezcan. Por ejemplo, Darling-Hammond $(2007,2010)$ critica la política norteamericana basada en estándares, señalando que los países con mejores resultados en las pruebas internacionales no usan las pruebas para hacer ranking o castigar a las escuelas, sino para apoyar el mejoramiento. Además, centran sus mediciones en el desarrollo del pensamiento crítico y la resolución de problemas, y focalizan sus esfuerzos en fortalecer la profesión docente y los procesos de formación. Los cambios educativos no ocurren si se utilizan los estándares solo como mecanismos de control; ellos deben ser parte de un sistema articulado de dispositivos que, en conjunto, mejoran los aprendizajes. El engranaje clave de este sistema son las capacidades

2 Por ejemplo, Baker (2004) distingue cuatro nociones de alineamiento -congruencia, correspondencia, puente y fuerza gravitacional- cada una con sus propios riesgos y mitigaciones. 
docentes (Darling-Hammond, 2001; Elmore, 2003; Bolívar, 2003; PISA, 2004).

En el ámbito de la formación de profesores, los estándares se relacionan con procesos de regulación que operan ya sea sobre los programas de formación o sobre las capacidades de los futuros profesores. En el primer caso, los mecanismos privilegiados son la aprobación de los programas de formación y la acreditación, en el segundo, los procesos de certificación y de registro de nuevos profesores (National Research Council, 2010).

Específicamente respecto de la formación de profesores, la experiencia muestra que los estándares no han repercutido en el mejoramiento educativo cuando se han impuesto externamente como dispositivos de control. En cambio, cuando los estándares son definidos por los profesores o por las mismas instituciones formadoras y estas se autoevalúan con un propósito de empoderamiento, son bien recibidos por los actores y pueden promover procesos de mejoramiento (Zuzovsky y Libman, 2006).

Los estándares son un dispositivo de regulación en estados descentralizados que operan, en los términos de Elliot (2002), como un Estado evaluador. En este modelo, el Estado adquiere servicios públicos, pero no es un proveedor directo de educación, y con el fin de asegurar la calidad del servicio establece estándares, financia y exige responsabilidades. Para ejercer su rol, el Estado debe evaluar el desempeño de las instituciones, influyendo a través de ello en la calidad educativa. La relación entre estado y mercado, y el rol específico que asume el Estado varía entre sistemas educacionales. En uno que confía más en el mercado, se minimiza la acción del Estado y la evaluación se utiliza como sistema de información para rankear las instituciones e informar la demanda, dejando que el mercado regule la calidad. Si se considera que el mercado informado no es un mecanismo suficiente -o desde otra perspectiva, pertinente- para regular la calidad, el Estado realiza otras acciones que pueden vincularse con sistemas de incentivos y control, o sistemas de apoyo y promoción, y distintas combinatorias de estos. Así, el Estado evaluador puede tomar diversas formas, es decir, puede ejercer una mayor o menor 
96 ESTÁNDARES Y REGULACIÓN DE CALIDAD DE LA FORMACIÓN DE PROFESORES: DISCUSIÓN DEL CASO CHILENO DESDE UNA PERSPECTIVA COMPARADA - Carmen Sotomayor, Jacqueline Gysling

intervención regulatoria y, consiguientemente, puede operar con una mayor o menor autonomía de las instituciones que ofrecen el servicio. El punto de la discusión es cuánta injerencia debe tener el Estado en la regulación de la calidad y cuánta confianza debe depositarse en las agencias del sistema para autorregular su calidad.

Al respecto, nuestro país figura en los análisis comparados internacionales en el extremo de la desregulación educativa, es decir, proveyendo un marco mínimo de actuación a las instituciones y actores, y entregando, mediante mediciones de aprendizaje, información a la demanda (Banco Mundial, 2007). Sin embargo, como es de conocimiento público, Chile está en proceso de instalar un sistema de regulación con mayor participación del Estado, tanto respecto de la totalidad del sistema, como específicamente en la formación de profesores. Dado que este es un proceso en curso y que existe una alta preocupación nacional por la calidad y equidad educativa, nos ha parecido un aporte a la reflexión examinar con detenimiento cuatro casos con una amplia trayectoria en regulación basada en estándares. Describiremos a continuación las medidas adoptadas y en curso en el país respecto a la formación de profesores.

\subsection{La regulación de la formación de profesores en Chile}

Desde la reforma del sistema de educación superior el año 1981, en nuestro país las instituciones de formación se han multiplicado y diversificado. Actualmente, existen alrededor de 60 instituciones que imparten carreras de pedagogía, que ofrecen programas de muy diferentes características, aplican distintos mecanismos de selección al ingreso e implementan sistemas de evaluación propios para medir el logro de sus objetivos. En los últimos años (particularmente desde el 2002 en adelante), la matrícula de educación ha aumentado en un 150\% (Panel de expertos para una educación de calidad, 2010) y el mayor incremento se ha producido en las instituciones que demandan menores requisitos académicos (Ceppe, 2011). Por otra parte, se cuenta actualmente con algunos resultados de mediciones de conocimientos y habilidades de estudiantes de pedagogía que muestran falencias importantes no solo en sus conocimientos disciplinarios, sino también pedagógicos. Es el caso de las recientes 
pruebas Inicia para estudiantes de educación de párvulos y básica (Belleï y Valenzuela, 2010; Ministerio de Educación, 2010) y del estudio internacional TEDs-M, que midió conocimientos disciplinarios y pedagógicos en matemática de estudiantes de pedagogía básica por egresar (Ávalos y Matus, 2010).

El sistema de educación superior en Chile opera en forma autónoma y liberalizada, y sólo recientemente se está avanzando en el establecimiento de mecanismos de regulación de la calidad a través de procesos de acreditación. La acreditación de carreras es voluntaria, salvo en el caso de las carreras de medicina y pedagogía que es obligatoria ${ }^{3}$. La obligatoriedad, en todo caso, sólo impide obtener recursos del Estado, es decir, una carrera de pedagogía que no se acredita, no significa que deba cerrarse, sino que debe autofinanciarse. Pese a la masiva acreditación de las carreras de educación, los informes de la Comisión Nacional de Acreditación (CNA) dan cuenta de la precariedad de muchos programas acreditados: particularmente la falta de barreras de ingreso y la poca orientación en las prácticas profesionales. Por otra parte, el sistema de acreditación adolece de una evaluación de la calidad de sus programas de estudio con referencia a estándares nacionales (Belleï y Valenzuela, 2010).

En este contexto, el año 2008 el Ministerio de Educación lanzó un programa nacional de mejoramiento de la formación docente denominado Inicia. Esta política contempla la elaboración de estándares y orientaciones curriculares que guíen los procesos de formación docente, así como la aplicación de una evaluación diagnóstica para determinar el logro de aprendizajes al finalizar sus procesos de formación. Se espera que los estándares y la evaluación de los estudiantes de pedagogía provean una información de base para que las universidades orienten mejor sus procesos formativos.

Estas dos líneas de acción se encuentran en desarrollo en el país. Por una parte, y bajo las orientaciones del Ministerio de Educación,

3 Las carreras que no se acreditan, sea porque no se presentan al proceso de acreditación o porque no logran ser acreditadas, no pueden acceder a ningún tipo de recursos otorgados directamente por el Estado o que cuenten con su garantía para el financiamiento de los estudios de sus nuevos alumnos (art. 27, ley 20.129). 
el $\mathrm{CIAE}^{4}$ de la Universidad de Chile y el $\mathrm{CEPPE}^{5}$ de la Universidad Católica, en colaboración con más de una veintena de universidades del país, han elaborado estándares de contenido (qué deben saber y saber hacer) para la formación de profesores de educación general básica en las áreas de lenguaje, matemática, ciencias sociales y naturales. Además, están en elaboración estándares de contenido para la formación de profesores de educación media en estas mismas áreas y para la formación de las educadoras de párvulos. En este desarrollo se ha recogido la definición de estándares elaborada por el programa de Fortalecimiento de la Formación Inicial Docente (Mineduc, 2001), que se implementó en la segunda mitad de los noventa y del Marco de la Buena Enseñanza (Mineduc, 2008) que, sin haber sido elaborados como tales, operan como los estándares que orientan la evaluación de los docentes en ejercicio.

Por otra parte, se aplicaron dos mediciones experimentales y voluntarias para evaluar las competencias de egreso de los estudiantes de pedagogía, realizadas por el Centro MIDE de la Pontificia Universidad Católica de Chile. En diciembre de 2010 se aplicó por tercera vez esta prueba y los resultados por primera vez se hicieron públicos en abril de 2011.

En el contexto de preocupación por fortalecer la profesión docente en el país, el nuevo gobierno que asumió en marzo de 2010 convocó a una comisión de expertos para que realizara recomendaciones al respecto. El panel de expertos para una educación de calidad (2010) entregó propuestas relevantes referidas al mejoramiento de los sistemas de acreditación de las carreras de pedagogía y de selección de alumnos, así como una propuesta de carrera docente en que se mejora la remuneración mínima inicial y luego a los cinco y diez años, procurando cerrar las brechas entre los salarios de profesores y los de otras profesiones.

Además recomendó, a partir de la prueba Inicia, instalar un examen de habilitación que fuera obligatorio aprobar para poder enseñar en la educación subvencionada. Algunas de estas

4 Centro de Investigación Avanzada en Educación.

5 Centro de Estudios de Políticas y Prácticas en Educación. 
recomendaciones han sido recogidas por la actual administración, aunque con variaciones importantes. Es el caso del proyecto de ley enviado en noviembre de 2010 al Congreso ( $N^{\circ}$ 370/358) que crea el "Examen de excelencia profesional docente". En este proyecto se regulariza la aplicación de la prueba Inicia como un mecanismo de incentivo y no como un examen de habilitación, ya que se define que este sería un examen voluntario de conocimientos disciplinarios que permiten el cumplimiento de los objetivos de aprendizaje definidos en las bases curriculares (art. 2). El proyecto señala que los contenidos del examen serán fijados por el Ministerio de Educación y aprobados por el Consejo Nacional de Educación (art. 3), y que sus resultados serán públicos por institución y por programa o carrera (art. 4). Asimismo, este proyecto propone una asignación de excelencia pedagógica inicial a los que hayan obtenido resultados destacados en este examen y que tengan un contrato de al menos 22 horas en algún establecimiento subvencionado (art. 7). Cabe destacar que en el proyecto de ley no se establece explícitamente que los contenidos de la prueba deban estar alineados a estándares.

Así como se han fijado medidas relacionadas con el egreso, también se han implementado incentivos para atraer a alumnos de mejor rendimiento a las carreras de pedagogía. Es el caso de las becas Elige Educar creadas por el Ministerio de Educación el año 2010, que buscan aumentar el número de postulantes con puntajes superiores a 600 y 700 puntos en la PSU. Para acceder a estas becas, los estudiantes deben matricularse en una institución y carrera acreditada y con puntaje de corte declarado por la institución de educación superior mayor a 500 puntos promedio PSU. Este requisito busca incentivar el mejoramiento de los procesos de formación.

Este rol un poco más activo del Estado en la formación de profesores se corresponde con la tendencia internacional en educación y con las demandas ciudadanas en Chile. En el ámbito internacional, incluyendo el latinoamericano, se ha producido en la última década un creciente interés por ampliar la regulación estatal del sistema educacional e instalar mecanismos de aseguramiento de la calidad, luego que los mecanismos de mercado, impuestos en los ochenta, no mostraran ser efectivos para asegurar una educación de calidad a 
100 ESTÁNDARES Y REGULACIÓN DE CALIDAD DE LA FORMACIÓN DE PROFESORES: DISCUSIÓN DEL CASO CHILENO DESDE UNA PERSPECTIVA COMPARADA - Carmen Sotomayor, Jacqueline Gysling

toda la población (Tedesco, 2007). En nuestro país, esta búsqueda se ha realizado sobre un cierto consenso de no volver al Estado docente anterior a los ochenta, que conducía y controlaba la totalidad del sistema, y ofrecía directamente el servicio educativo. En cambio, se ha definido avanzar hacia un sistema que mantenga la provisión mixta, pero con una mayor participación regulatoria del Estado (Comisión Asesora Presidencial para la Calidad de la Educación, 2006).

La discusión sobre la acción del Estado en educación, que tuvo una coyuntura emblemática con el movimiento estudiantil de 2006, sigue vigente y muestra de ello es el vigoroso movimiento de los estudiantes chilenos en 2011. Está por verse la forma que adopte definitivamente la regulación estatal luego de estos eventos, pero por el momento los términos del debate hablan de la creación de una superintendencia para el nivel de educación superior que fiscalice la prohibición legal del lucro en el nivel universitario y de la instalación del sistema de aseguramiento de la calidad diseñado en la Ley General de Educación para el sistema escolar en 2009.

Entre las políticas que se discuten actualmente se incluye también la formación de profesores. En la propuesta del Ministerio de Educación al movimiento estudiantil (agosto 2011) se afirma que se entregarán estándares orientadores a las facultades de pedagogía, se promoverán convenios de desempeño con estas y se hace presente que el Ejecutivo ya ha ingresado al Congreso un proyecto de ley que establece la obligatoriedad de la prueba Inicia para los egresados de pedagogía, lo que supondría cambiar el proyecto de ley original que establece que esta prueba es voluntaria, como ya se ha dicho.

\subsection{El estudio}

El estudio realizado consistió en analizar en cuatro casos cómo operan los estándares como dispositivo de un sistema conformado por regulaciones, procedimientos y agencias interrelacionados. Tomando en cuenta el estado de la discusión en Chile, ha resultado de particular interés analizar:

a) Las características de los estándares. Si estos son generales o específicos, es decir, si definen las competencias o los saberes que deben tener todos los docentes sin distinción de nivel o 
disciplina, o si los estándares son específicos para cada tipo de profesor. En particular, nos ha interesado determinar si hay definiciones sobre los saberes que se deben lograr en el ámbito de las disciplinas de formación.

b) La relación entre los estándares de formación inicial, que describen las competencias al egreso del proceso de formación, y los estándares profesionales, que describen las competencias que se espera de un profesor en ejercicio de su profesión.

c) La acción reguladora del Estado a partir de los estándares; especialmente, cómo operan los procesos de acreditación de programas de formación y de certificación de nuevos profesores.

d) La institucionalidad del Estado para ejercer su acción reguladora de la formación de profesores.

Este artículo expone los resultados de la investigación financiada por el Consejo Nacional de Educación: "Revisión comparada de los sistemas y políticas de mejoramiento de la formación de profesores guiadas por estándares en países anglosajones: lecciones para Chile". La investigación se basó en información disponible en la web, principalmente en los sitios oficiales vinculados con la formación de profesores, y en testimonios sobre la implementación de los estándares en una universidad de cada estado o provincia analizados. Estos testimonios se recolectaron mediante entrevistas a las autoridades correspondientes, realizadas por estudiantes chilenas que cursan posgrados en educación en esas mismas universidades.

Los casos que aborda el estudio son el estado de Victoria, en Australia; la provincia de Quebec, en Canadá; el estado de Nueva York, en Estados Unidos, e Inglaterra en el Reino Unido. En los tres primeros casos se consideró un estado o provincia del país, pero también se caracteriza el sistema en general para describir las relaciones entre el nivel nacional y el estadual. En el caso de Inglaterra, administrada por el gobierno central del país, no se hace esta distinción. Los casos se determinaron considerando que, al igual que Chile, tienen un sistema de formación de profesores mixto, donde distintas instituciones de educación superior públicas y privadas realizan la preparación de los futuros profesores. Por esta misma razón, estos países han sido un 
102 ESTÁNDARES Y REGULACIÓN DE CALIDAD DE LA FORMACIÓN DE PROFESORES: DISCUSIÓN DEL CASO CHILENO DESDE UNA PERSPECTIVA COMPARADA - Carmen Sotomayor, Jacqueline Gysling

referente de las políticas educativas de Chile en las últimas décadas. Además, resultan particularmente interesantes por las siguientes razones:

- En ellos existen agencias estatales especializadas y una política explícita del estado orientada a regular la calidad de la formación docente basada en estándares.

- Tienen una experiencia acumulada en regulación de la formación de profesores, lo que permite observar el desarrollo del sistema y las modificaciones incorporadas a partir de la experiencia.

- Son diferentes entre sí. Unos sistemas presentan una mayor acción reguladora del Estado y otros, una menor. Unos tienen sistemas de pruebas como requisito para la certificación y otros no. En el caso de Canadá se consideró el estado de Quebec para acercarse a una experiencia con influencia francófona.

Finalmente, un criterio operativo fue el idioma del país, requisito para poder acceder a la documentación oficial mediante la web.

\section{Cuatro casos de política de formación de profesores basadas en estándares}

Los sistemas de formación docente estudiados son complejos y presentan particularidades en cada caso, que los distinguen. Una primera constatación es que en los cuatro casos examinados no hay una manera única de definir estándares ni de implementarlos en el sistema. Una segunda, es que es un campo totalmente dinámico, algunos hablan de candente, en el sentido de que hay mucha atención, preocupación y elaboración en curso. En tres de los cuatro casos encontramos definiciones de estándares del año 2011 y cambios en las regulaciones. Este dinamismo es expresivo de la búsqueda, no concluida, de mejores definiciones, ya que no existe evidencia sistemática que evalúe el impacto de la implementación de sistemas de estándares en la calidad de los procesos de formación. Una tercera observación es que en todos ellos hay una acción decidida del estado para asegurar la calidad. La voluntad del estado por actuar sobre la calidad se expresa en la existencia de entidades estatales especializadas en resguardar la calidad 
de la profesión docente, dependientes de las máximas autoridades de educación. Estas agencias se ocupan de varias funciones: definen los estándares, acreditan, registran y/o certifican a nuevos profesores.

A continuación, describiremos comparativamente los cuatro casos estudiados, no obstante, antes daremos una imagen global de las particularidades de cada sistema y del estado de la discusión sobre regulación de formación de profesores en cada uno de ellos. La información detallada caso a caso se ha sintetizado en la tabla 1 .

\section{Victoria - Australia}

Australia hasta 2010 tenía una regulación descentralizada, es decir, cada estado y territorio del país tenía su propia normativa sobre formación docente. A partir de 2011, está implementando un sistema nacional de regulación de la profesión docente que reproduce a escala nacional lo que existía en algunos estados, entre ellos, el de Victoria. En este estado existe una agencia estatal especializada, el Victorian Institute of Teaching (VIT), creada en 2001, que controla los procesos de acreditación de los programas de formación y certificación de profesores. Esta institución estableció los estándares con los cuales se evalúan los procesos de formación y se realiza la certificación de profesores. Los estándares son ocho, son generales; es decir, no hacen distinción por nivel o asignatura del profesor e incluyen tres ámbitos: conocimiento profesional, habilidades prácticas y compromiso profesional. Al término de los estudios universitarios, los nuevos profesionales deben postular a un registro provisional, que tiene una duración de dos años. Para postular al registro permanente se exige haber ejercido en el sistema 80 días y entregar evidencia del cumplimiento de los estándares. Esta evidencia la entrega el director de la escuela donde el profesor trabajó.

Al comenzar a funcionar el sistema nacional de regulación de la profesión deja de funcionar el VIT. La agencia nacional se denomina Australian Institute for Teaching and School Leadership (AITSL). Los estándares nacionales han sido objeto de una amplia consulta en todo el país, para llegar a una formulación similar a la de Victoria, que tiene siete estándares generales, organizados en los mismos ámbitos. Una novedad del sistema nacional es la organización de 
104 ESTÁNDARES Y REGULACIÓN DE CALIDAD DE LA FORMACIÓN DE PROFESORES: DISCUSIÓN DEL CASO CHILENO DESDE UNA PERSPECTIVA COMPARADA - Carmen Sotomayor, Jacqueline Gysling

los estándares de formación inicial y profesionales en una gradiente que busca impulsar el establecimiento de incentivos para favorecer la retención de los buenos profesores, ya que se ha observado que no es suficiente regular la calidad de los procesos de formación para mejorar las capacidades del cuerpo docente.

\section{Quebec - Canadá}

Canadá tiene un sistema descentralizado de administración de la educación, es así que cada provincia presenta particularidades. El sistema de regulación en Quebec se destaca por su estabilidad y confianza en las instituciones formadoras, lo cual se sostiene en procesos de acreditación rigurosos que son obligatorios. Existe una institución especializada que regula todo el proceso, la Dirección de Formación y Titulación de Profesionales de la Educación, y una agencia autónoma, el Comité de Consentimiento de Programas de Formación de Educación (CAPFE, por sus siglas en francés), que realiza el proceso de acreditación y lo reporta a la dirección de formación del Ministerio de Educación de la provincia. En cuanto a estándares, tienen lo que denominan un "referencial de competencias", definido en el 2001, que establece 12 competencias profesionales, para cada una de las cuales se dan indicadores que corresponden a lo que debe lograr un profesional recién egresado. Este referencial de competencias se destaca por el tipo de profesional que perfila, ya que incluye competencias críticas y éticas, ausentes en las definiciones de los otros países analizados. La evaluación del logro de las competencias se realiza como parte del proceso de formación, lo cual se supervisa mediante el proceso de acreditación.

\section{Nueva York - Estados Unidos}

Estados Unidos tiene una administración descentralizada de su sistema educativo, aunque existen definiciones de estándares y agencias acreditadoras que tienen carácter nacional y actúan en distintos estados. En el ámbito nacional, son particularmente relevantes los estándares del Interstate New Teacher Assessment and Support Consortium (INSTASC), que definió los primeros estándares de formación de profesores en 1987. Estos fueron reformulados en 2010, como estándares profesionales. También, son relevantes los estándares 
de la agencia nacional de acreditación de formación de profesores, el National Council for Accreditation of Teacher Education (NCATE), que se encuentra en proceso de fusión con la otra agencia acreditadora de programas de formación denominada Teacher Education Accreditation Council (TEAC). Ambas han definido una nueva orientación para la formación de profesores con énfasis en la práctica. También son relevantes los estándares de excelencia profesional del National Board for Professional Teaching Standards (NBPTS).

En Nueva York existe desde 2000 una política de control de la profesión docente, que comenzó por exigir que todos los profesores se registren en una entidad creada para tal fin, la Office of Teaching Initiatives. Para ser registrados, los profesores deben cumplir una serie de requerimientos, entre ellos, haber obtenido un título en una institución acreditada por una agencia nacional (NCATE o TEAC) o la agencia estadual (Regents Accreditation of Teacher Education - RATE) y aprobar una serie de exámenes. Estos son administrados por otra repartición denominada New York State Teacher Certifications Examination (NYSTCE). Los exámenes son específicos por nivel y disciplina. Existe una certificación inicial al egreso del proceso de formación y una certificación permanente, que exige un año de inducción y tres años de ejercicio profesional. El proceso de certificación no se vincula directamente con los procesos de formación, ni tiene consecuencias para estos, sino que se lo entiende más bien como un proceso personal de los candidatos que el estado apoya mediante mecanismos de formación continua.

En Nueva York, tanto las autoridades como la universidad estudiada, operan con varios estándares como referentes. No obstante, en 2011 el departamento de educación estadual dio a conocer un conjunto de estándares que regirán la certificación a partir de 2013. Ellos buscan establecer una regulación más clara y centrada en el desempeño profesional por sobre los conocimientos. En 2013 se modificarán los requerimientos para obtener la certificación, que incluye entre las exigencias entregar un portafolio con evidencias de desempeño. Los exámenes se mantienen, pero se anuncia que se modificarán. 
106 ESTÁNDARES Y REGULACIÓN DE CALIDAD DE LA FORMACIÓN DE PROFESORES: DISCUSIÓN DEL CASO CHILENO DESDE UNA PERSPECTIVA COMPARADA - Carmen Sotomayor, Jacqueline Gysling

Tanto en Estados Unidos como en Nueva York ha habido una serie de nuevas definiciones de estándares, que parecen apuntar hacia un fortalecimiento de la acreditación y un mayor énfasis en los desempeños prácticos.

\section{Inglaterra - Reino Unido}

Inglaterra va en camino de simplificar un sistema que comenzó en los ochenta con una reforma profunda al sistema de formación de profesores. En esa época se autorizó a consorcios de escuelas a actuar como instituciones formadoras, además, se crearon una serie de instituciones estatales para operar la regulación del sistema y otorgar el Qualified Teacher Status (QTS), que se exige para ejercer en la educación pública. Además, en los noventa se llegó a establecer un currículo único de formación de profesores. Las agencias que se crearon en el proceso fueron la Training and Development Agency (TDA) que define los estándares y administra la aprobación de programas de formación, el General Teaching Council (GTC), que lleva el registro de profesores y otorga el QTS, y la Office for Standards in Education (Ofsted), que realiza la supervisión de instituciones escolares y de formación de profesores.

La resistencia a esta reforma y la evaluación de su implementación han incidido en la incorporación de sucesivos cambios al diseño original. En 2002 se eliminó la idea de un currículo de formación único y se establecieron 33 estándares. Luego, en 2008 estos estándares se articularon en un sistema graduado con los estándares profesionales. El cambio más reciente, dado a conocer en junio de 2011, redujo a ocho los 33 estándares que estaban vigentes y los unificó con el código de comportamiento ético, incluyendo tres estándares más. También, eliminaron un nivel de la gradación que distinguía entre los conocimientos, habilidades y atributos que debía tener un estudiante al egreso del proceso de formación y los que debía tener el profesor al ingresar al ejercicio profesional, luego del proceso de inducción. Al mismo tiempo, se han anunciado transformaciones en las agencias del sistema, que incluye el cierre de la General Teaching Council (GTC). Es decir, en el momento actual, el más centralizado de los casos estudiados va en camino de simplificación de un modelo que las actuales autoridades han calificado como poco eficaz. 


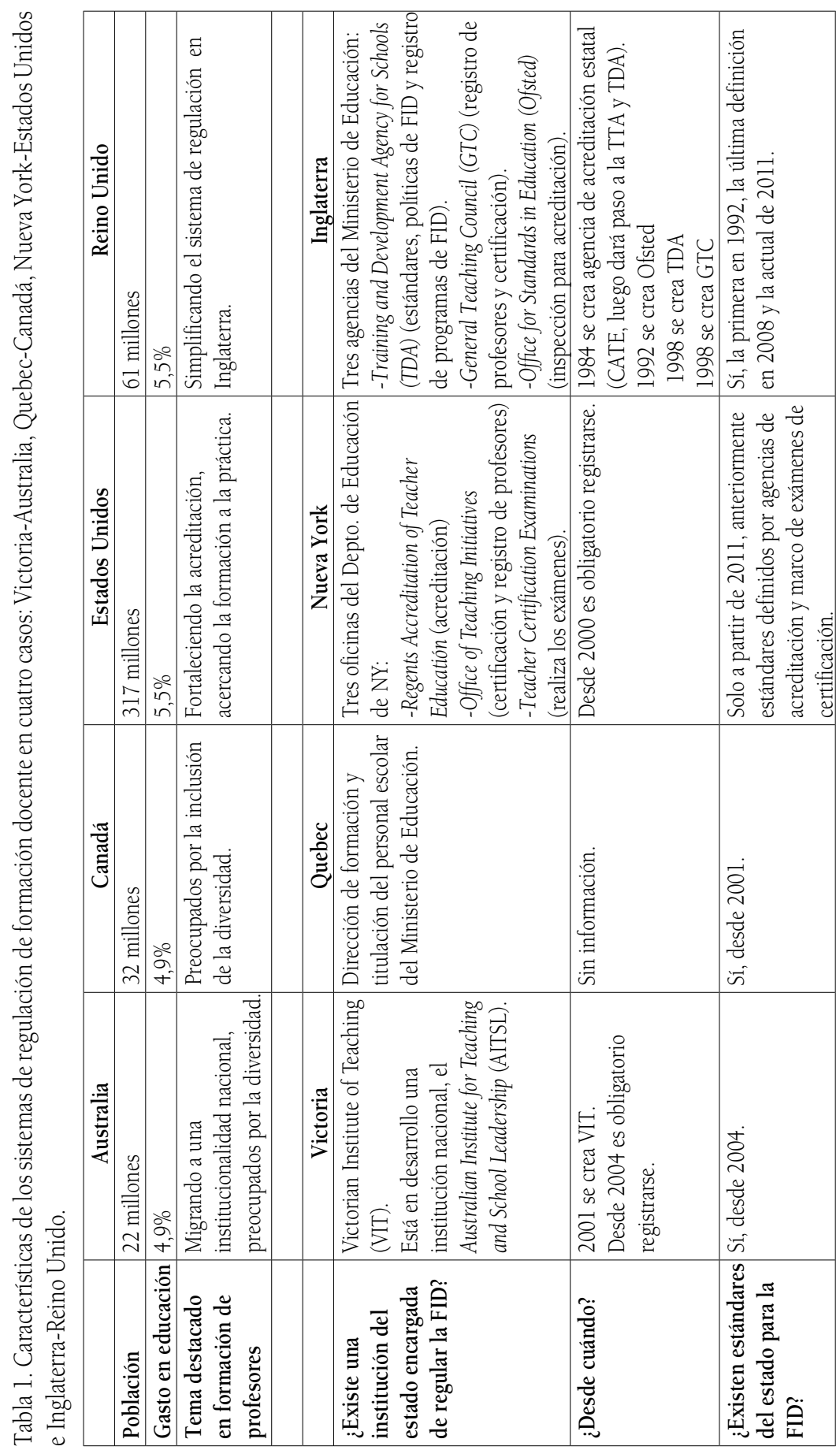


108 ESTÁNDARES Y REGULACIÓN DE CALIDAD DE LA FORMACIÓN DE PROFESORES: DISCUSIÓN DEL CASO CHILENO DESDE UNA PERSPECTIVA COMPARADA - Carmen Sotomayor, Jacqueline Gysling

\begin{tabular}{|c|c|c|c|c|c|}
\hline 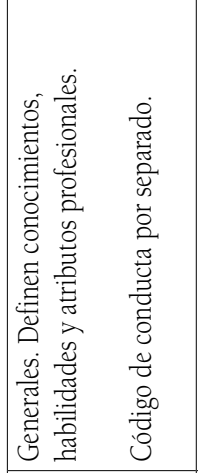 & 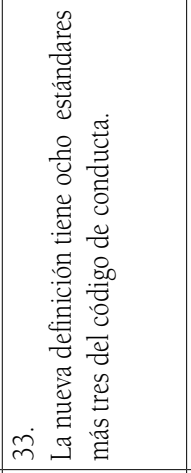 & $\dot{z}$ & 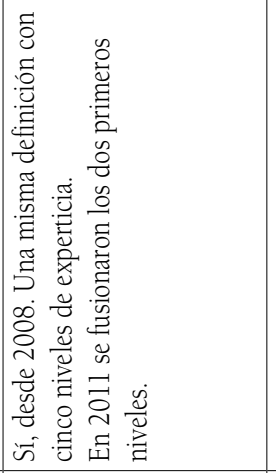 & 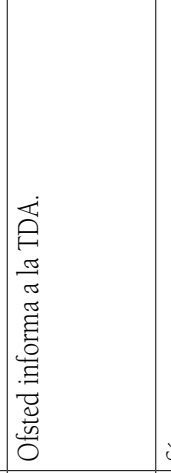 & inj \\
\hline 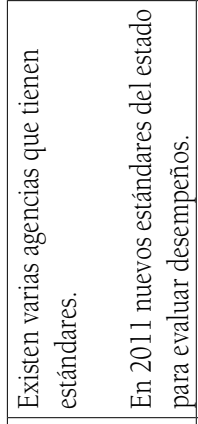 & 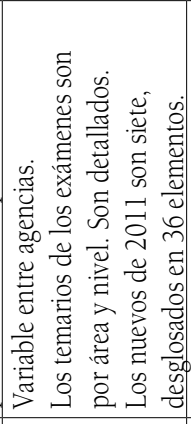 & 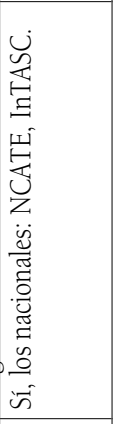 & 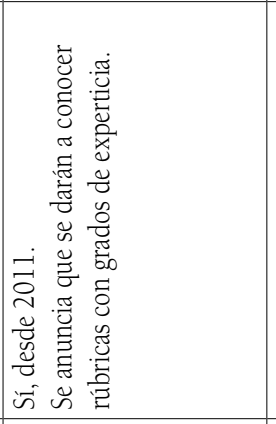 & 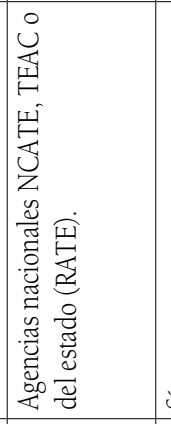 & ఉં \\
\hline 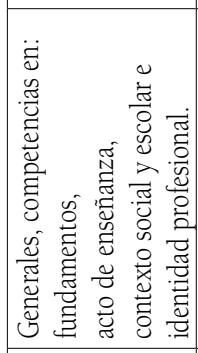 & $\underset{\sim}{\sim}$ & $\dot{z}$ & 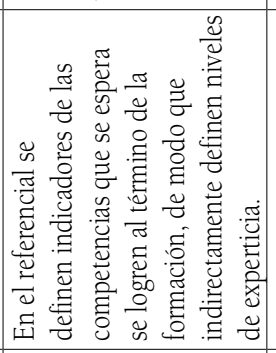 & 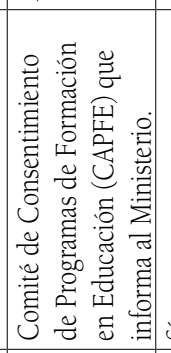 & 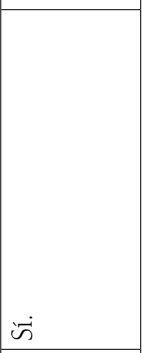 \\
\hline 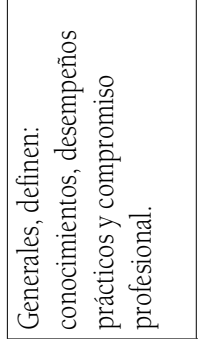 & $\infty$ & $\dot{2}$ & 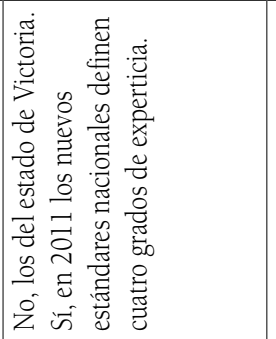 & 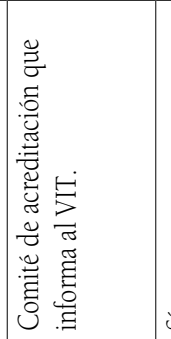 & in \\
\hline 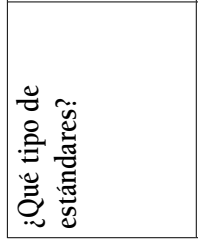 & 苟 & 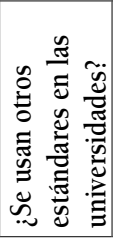 & 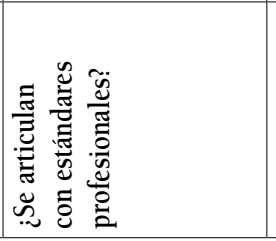 & 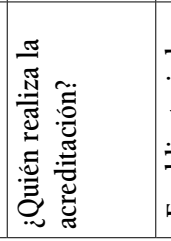 & 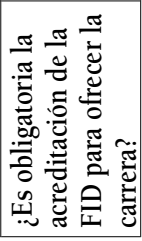 \\
\hline
\end{tabular}




\begin{tabular}{|c|c|c|c|c|}
\hline 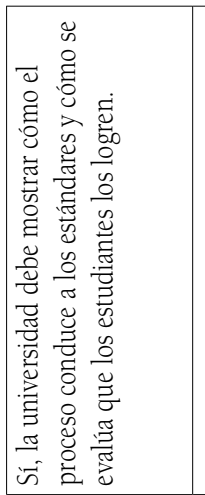 & & 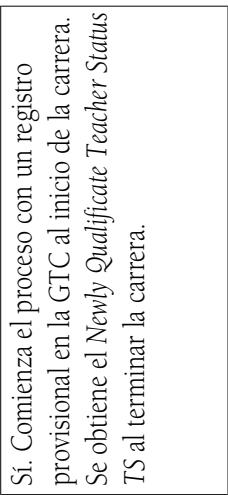 & 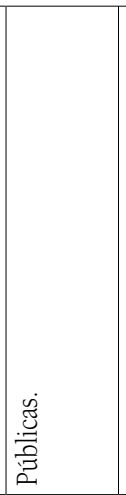 & 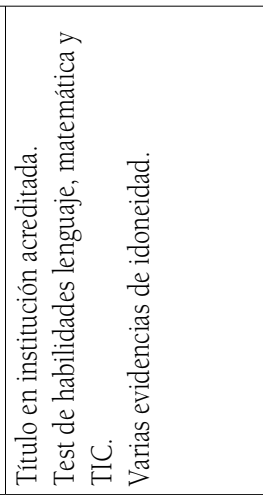 \\
\hline 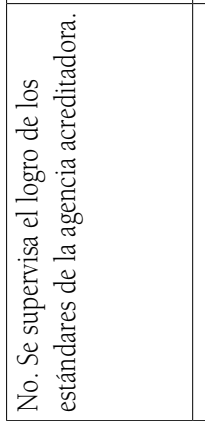 & 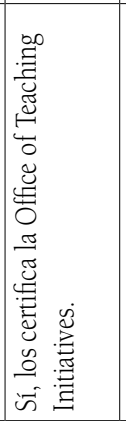 & 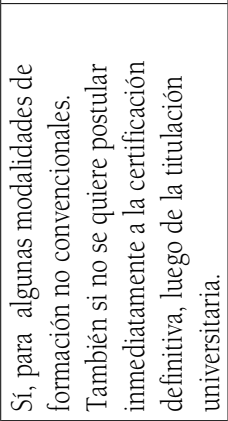 & 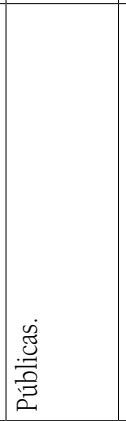 & 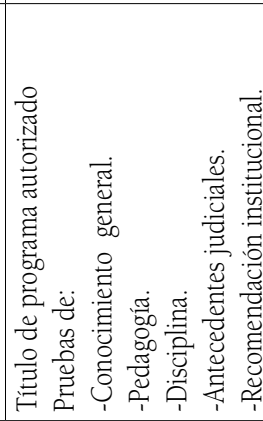 \\
\hline 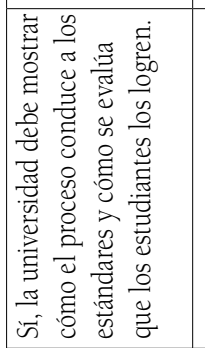 & 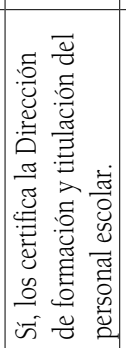 & & 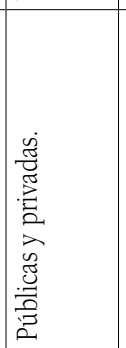 & 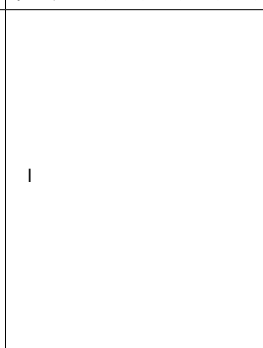 \\
\hline 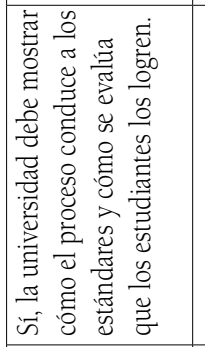 & 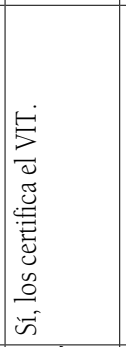 & 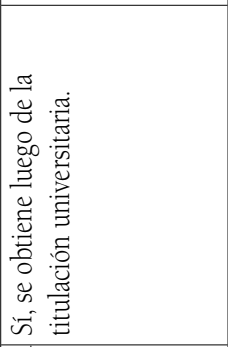 & 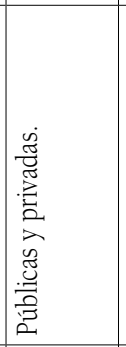 & 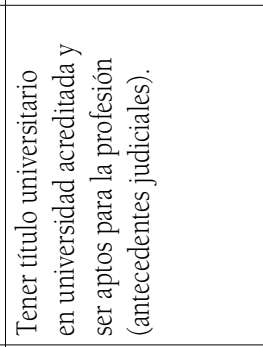 \\
\hline 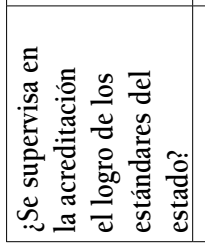 & 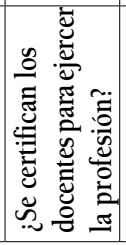 & 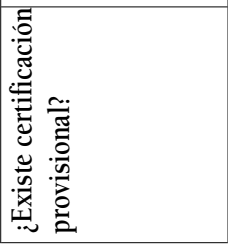 & 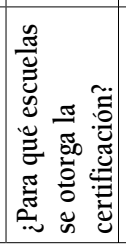 & 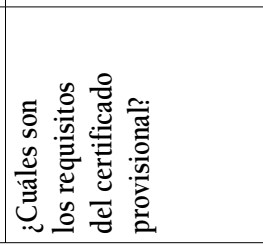 \\
\hline
\end{tabular}


110 ESTÁNDARES Y REGULACIÓN DE CALIDAD DE LA FORMACIÓN DE PROFESORES: DISCUSIÓN DEL CASO CHILENO DESDE UNA PERSPECTIVA COMPARADA - Carmen Sotomayor, Jacqueline Gysling

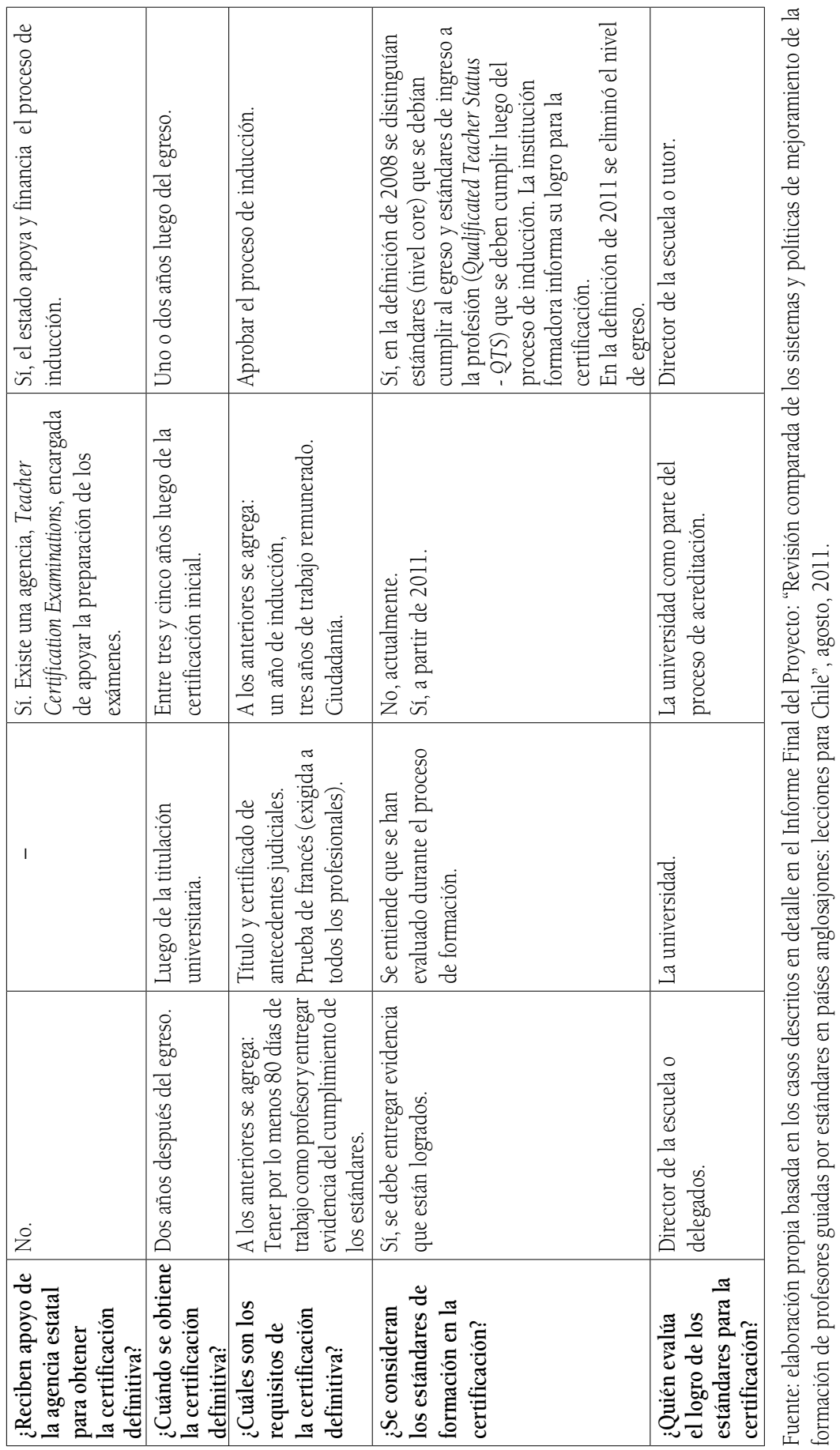




\subsection{La acreditación}

En los cuatro casos analizados, los estándares operan fundamentalmente mediante los procesos de acreditación. En Australia, Canadá e Inglaterra, las universidades deben acreditar sus programas de formación y se les exige que muestren cómo cada curso de la formación conduce al logro de los estándares. En Australia, Estados Unidos e Inglaterra hay agencias gubernamentales nacionales o estaduales específicas para la formación de profesores que realizan la certificación y control de los programas de formación. En el caso de Canadá, estas son agencias originadas desde el Ministerio de Educación de cada provincia, pero su funcionamiento es autónomo y en ellas participan, de manera colegiada, académicos y profesionales del medio escolar.

Existe bastante diferencia en la forma en que se realizan los procesos de acreditación. En Estados Unidos existe una agencia predominante de acreditación de los programas de formación docente, el National Council for Accreditation Teacher Education (NCATE), que acumula críticas por lo burocratizado y engorroso de sus procesos. Actualmente, el NCATE se encuentra en proceso de fusión con el Teacher Education Accreditation Council (TEAC), agencia que surgió para contrarrestar la inercia de NCATE. En Canadá, la acreditación es estadual y se realiza por una agencia autónoma que reporta a la agencia del estado. En Australia, que también operaba descentralizadamente, se ha creado una agencia del gobierno federal que será la entidad acreditadora nacional (Australian Institute for Teaching and School Leadership - AITSL). En Inglaterra se creó, en los noventa, una agencia estatal de regulación de la profesión docente (Teacher Training Agency, luego Training and Development Agency for Schools) y un servicio de inspección del cumplimiento de los estándares (Office for Standards in Education - Ofsted).

En los procesos de acreditación se supervisa el cumplimiento de los estándares, pero son las mismas instituciones que evalúan el cumplimiento de los mismos. Para ello son definidos diversos procedimientos, equipos y comisiones especializadas internas que tienen gran importancia para las instituciones formadoras que siguen una lógica de mejoramiento continuo de sus programas de formación. En el proceso de acreditación se revisa cómo la universidad realiza esta 
112 ESTÁNDARES Y REGULACIÓN DE CALIDAD DE LA FORMACIÓN DE PROFESORES: DISCUSIÓN DEL CASO CHILENO DESDE UNA PERSPECTIVA COMPARADA - Carmen Sotomayor, Jacqueline Gysling

evaluación, pero no existe en ninguno de los cuatro casos analizados una evaluación externa de los conocimientos de los egresados. Las evaluaciones de conocimientos están relacionadas con los procesos de habilitación para el ejercicio profesional, pero no están vinculadas a procesos de acreditación.

Las cuatro universidades analizadas en la investigación son acreditadas y organizan sus procesos de formación considerando los estándares que les exigen para la acreditación. Pero todas ellas consideran que los estándares les definen un piso mínimo y que sus procesos de formación son más amplios, ya que fomentan el desarrollo de un profesional reflexivo capaz de observar, analizar críticamente y mejorar su práctica, así como seguir aprendiendo a lo largo de su carrera profesional.

\subsection{La certificación}

Al egresar de los programas de formación, los estudiantes reciben una certificación provisional para ejercer la docencia. Luego de uno, dos o cinco años de ejercicio deben optar por una certificación permanente. En ese momento, a los profesores novicios se les exige demostrar que cumplen los estándares. Una excepción es Quebec, ya que las universidades incluyen dentro del proceso de formación el ejercicio práctico, de modo que la titulación incluye la experiencia.

El sistema de evaluación que contempla más requerimientos es el de Nueva York, los postulantes deben rendir una serie de exámenes además de mostrar solvencia práctica por medio de videos e informes del director del establecimiento. Los ingleses les siguen en cuanto a requerimientos, pero sus exámenes son de habilidades básicas y el fuerte de la evaluación son los informes de desempeño. En el caso de Australia no hay exámenes, sino informes de desempeño. En Canadá solo existe una prueba que evalúa el dominio de la lengua materna (inglés o francés) y el título de la institución formadora es un antecedente suficiente.

Entre la certificación provisoria y la definitiva, en Inglaterra se realiza un proceso de acompañamiento de los profesores novicios por mentores, profesores destacados de mayor experiencia y que están en 
las escuelas para complementar el proceso formativo. En el caso de Victoria-Australia no existe este proceso de acompañamiento, sino que los profesores comienzan su ejercicio profesional y luego de un año postulan a la certificación definitiva que tiene como evidencia principal el reporte del director de la escuela donde ha trabajado el postulante. En Nueva York para apoyar la certificación definitiva existe un programa del gobierno estadual, que mantiene un sitio web con materiales para preparar los exámenes. En Canadá el proceso de inducción queda a criterio de las escuelas y no se exige para obtener la certificación.

La certificación no se usa para juzgar la calidad de los procesos de formación, el caso extremo en esto es el de Quebec, donde la certificación asume que la calidad de la formación es responsabilidad de la universidad. En tanto, en Nueva York, Inglaterra y Victoria la distancia entre la certificación provisoria y la definitiva define una transición que libera a la universidad de la responsabilidad sobre la certificación. Esta depende del nuevo profesor y de cómo se desenvuelva en la práctica o en los exámenes de habilitación. Los exámenes de habilitación más exigentes son los del estado de Nueva York, que incluyen pruebas de conocimientos del área de especialización. Los exámenes de Inglaterra, en tanto, son de habilidades básicas lingüísticas, matemática y manejo de TIC.

La certificación en el caso de Victoria-Australia, de QuebecCanadá y de Nueva York-Estados Unidos tiene un alcance regional, lo que obstaculiza la movilidad de profesores en el país. En el caso de Victoria y Quebec la certificación habilita para desempeñarse en cualquier escuela pública o privada, en tanto, en Nueva York e Inglaterra es un requerimiento para desempeñarse en las escuelas públicas, no así en las privadas.

Luego de la certificación definitiva que desemboca en el registro de profesores, en todos los casos estudiados las certificaciones profesionales durante la carrera docente son voluntarias y se orientan a destacar a profesores de excelencia. En Australia este sistema se ha organizado recientemente, considerando incentivos salariales para los profesores que obtienen la certificación de excelencia. Esto 
114 ESTÁNDARES Y REGULACIÓN DE CALIDAD DE LA FORMACIÓN DE PROFESORES: DISCUSIÓN DEL CASO CHILENO DESDE UNA PERSPECTIVA COMPARADA - Carmen Sotomayor, Jacqueline Gysling

es considerado relevante para retener a los mejores docentes en las escuelas que atienden a alumnos de menor nivel socioeconómico y para elevar la valoración de la profesión en relación a otros profesionales.

\subsection{Los estándares}

En Estados Unidos no se hace una distinción entre estándares para profesores graduados y estándares profesionales para profesores en ejercicio. Este país, pionero en la definición de estándares de formación inicial, abandonó esta distinción, señalando que las diferencias no son sustantivas, sino que son de grado y relativas a la flexibilidad que muestra un profesor experto respecto a uno novicio. No obstante, sí definen estándares para la excelencia profesional (National Board for Professional Teacher Standards - NBPTS).

En Inglaterra y Australia, en cambio, se han definido estándares reconociendo niveles que parten por el nivel graduado (egresado de pedagogía) y luego se identifican niveles de desempeño profesional que operan como certificaciones voluntarias a las que postulan los profesores en ejercicio. En ambos casos, los estándares se han diseñado para impulsar el mejoramiento profesional de los docentes. En Australia, este mejoramiento se está diseñando como un sistema voluntario de incentivos salariales. A pesar de que en Estados Unidos, como se señaló, los estándares de formación y profesionales se han unificado en una sola definición, en el estado de Nueva York se ha anunciado que van a elaborar rúbricas que permitan definir una gradiente profesional y asociar incentivos al mejor desempeño. Inglaterra está en revisión de sus estándares graduados y recientemente (junio de 2011) ha dado a conocer una unificación de los dos primeros niveles (preinducción y postinducción), y anuncia para el 2012 la revisión de los niveles de desempeño profesional.

En cuanto al tipo de estándares definidos, se observa también variaciones importantes entre países. Considerando solamente el número de estándares se observan diferencias entre propuestas más sintéticas y definiciones más detalladas. En Australia se definen 7 estándares en la propuesta nacional y 8 en el estado de Victoria. 
En Canadá varían entre 6 estándares en el estado de Alberta, 8 en British Columbia, 9 en Ontario y 12 en el referencial de competencias de Quebec. En Estados Unidos existe variedad de definiciones, los estándares del InTASC son diez. Todos los casos mencionados corresponden a propuestas sintéticas. Por otro lado, las propuestas detalladas serían las de Inglaterra que define 33 estándares y que está migrando a una versión más sintética de 11 estándares; la reciente propuesta de Nueva York que define 7 estándares, pero que los desarrolla en 36 más específicos; y la propuesta de excelencia profesional del NBPTS, que define 5 estándares, pero complementa esta definición con estándares detallados por especialidad y nivel, constituyéndose en la propuesta más específica de estándares disciplinarios. En las entrevistas en Australia se planteó que en la propuesta nacional de estándares se optó por una versión sintética, pero el modelo que aspiran desarrollar es el del NBPTS, ya que se considera muy relevante desarrollar estándares que distingan las diferentes especialidades o disciplinas.

Un caso aparte lo constituyen los estándares de acreditación de NCATE, Estados Unidos, que consisten en 6 estándares, de ellos solo uno se refiere a lo que deben saber y saber hacer los profesores y los otros 5 son estándares institucionales relacionados con la calidad de los procesos de formación.

La visión de los entrevistados respecto al carácter sintético o detallado de los estándares es que prefieren los estándares sintéticos, ya que esto les otorga una mayor flexibilidad. Mientras más detallados, más engorrosa es su utilización y más restringidos se sienten para desarrollar sus propios énfasis en los procesos de formación.

En cuanto a la influencia de los estándares en los procesos formativos, la mayoría de los entrevistados de los distintos países coincide en que tienen un impacto en la orientación de los contenidos de los programas y también en los sistemas de monitoreo o evaluación interna de los mismos. En este sentido, otorgan un piso mínimo común que todas las universidades deben respetar. Menos importancia les atribuyen los entrevistados de Estados Unidos, para quienes el carácter 
116 ESTÁNDARES Y REGULACIÓN DE CALIDAD DE LA FORMACIÓN DE PROFESORES: DISCUSIÓN DEL CASO CHILENO DESDE UNA PERSPECTIVA COMPARADA - Carmen Sotomayor, Jacqueline Gysling

demasiado general y burocrático de los estándares de acreditación no significa un aporte a los procesos formativos.

Otra dimensión relevante de analizar son los contenidos de los estándares. Un elemento común es que todas las propuestas dan una visión completa de la profesión, destacando conocimientos y habilidades, o competencias que se consideran fundamentales. En términos de las áreas consideradas, también se observa bastante correspondencia al incluir estándares referidos a enseñanza, compromiso profesional y consideración contextual de los alumnos. El referencial de competencias de Quebec es el único que plantea explícitamente la dimensión crítica, al señalar que el profesor debe "actuar como un profesional crítico, intérprete de saberes o de la cultura durante el ejercicio de sus funciones". Ingleses y australianos, por su parte, dan mayor importancia al entendimiento del proceso de aprendizaje de los alumnos.

\section{Discusión y reflexiones para Chile}

Frente a este contexto brevemente esbozado, ¿qué rescatar de la experiencia de los países estudiados? ¿Qué nuevos elementos introducir para el análisis y discusión de nuestras políticas de formación docente?

\subsection{En relación con las instituciones y programas de formación de profesores}

La institucionalidad en la que se realiza la formación de profesores en los casos estudiados radica principalmente en las facultades de educación; sin embargo, en Inglaterra y en Estados Unidos existen diversas rutas alternativas de ingreso a la docencia. Incluso en Inglaterra consorcios de escuelas autorizados pueden realizar programas de formación.

Todas las universidades analizadas atraen buenos candidatos a los programas de formación de profesores, ya sea porque son universidades de alto prestigio o, en el caso de Canadá, porque la profesión tiene una alta valoración social. El gran interés por estudiar 
pedagogía en las instituciones investigadas hace que estas tengan sistemas de selección, que incluyen los antecedentes académicos de los postulantes, ya sean escolares o post-secundarios, siendo exigentes en sus requisitos. Existen algunos otros criterios para seleccionar a los postulantes que tienen relación con su calidad ética y capacidad para trabajar con niños.

Aun cuando las instituciones incluidas en el estudio son capaces de atraer postulantes con buenos antecedentes, existe preocupación por la capacidad limitada de hacerlo si las remuneraciones y condiciones de trabajo se mantienen inalteradas. Es el caso de Australia, que está desarrollando estándares y sistemas de incentivos ligados al desempeño para atraer a buenos estudiantes a la profesión y retener a los buenos profesores. Algo similar ocurre en Canadá, que ha invertido recursos significativos en políticas de mentoría, reducción de alumnos por curso y más apoyo de especialistas. Estos elementos, ya recomendados por el panel de expertos para una educación de calidad (2010), en particular la implementación de una carrera docente, están todavía pendientes en el escenario nacional.

En todos los países estudiados los modelos formativos ponen su foco en la práctica, y los estándares han jugado un papel importante en promover este énfasis. Esto quiere decir que el trabajo en la escuela es parte fundamental de la formación de los futuros profesores y que este es cuidadosamente planificado y monitoreado sin dejar nada al azar. En Inglaterra esta preocupación dio origen a modelos de formación de profesores impartidos por las propias escuelas organizadas en consorcios. Pero su manifestación más habitual es que la formación se mantenga en las universidades. Sin embargo, los tiempos de trabajo en las escuelas, tanto de los formadores como de los estudiantes, son proporcionalmente altos y los problemas escolares son abordados como problemas interesantes para investigar. También es frecuente que los supervisores de práctica sean profesores experimentados de los mismos colegios, que son contratados por un periodo determinado (uno o dos años) para formar parte del equipo de formadores de la facultad de educación y trabajar en colectivos en que la teoría y la práctica están íntimamente imbricadas. 
118 ESTÁNDARES Y REGULACIÓN DE CALIDAD DE LA FORMACIÓN DE PROFESORES: DISCUSIÓN DEL CASO CHILENO DESDE UNA PERSPECTIVA COMPARADA - Carmen Sotomayor, Jacqueline Gysling

La preocupación por las prácticas viene instalándose en Chile desde el programa de Fortalecimiento de la Formación Inicial Docente (FFID) en los años 1997-2002. La mayoría de los programas de formación de profesores en nuestro país cuenta en este momento con varias prácticas durante la trayectoria formativa. De las experiencias internacionales estudiadas, tal vez lo más relevante sea el valor académico que dan a estas prácticas. Es la escuela el lugar formativo por excelencia y es también la escuela la fuente principal del conocimiento pedagógico, propio de la profesión docente. Esto tiene implicancias concretas en la selección y forma de trabajo del cuerpo académico: equipos mixtos entre especialistas disciplinarios y profesores con experiencia en enseñanza escolar y desplazamiento físico a la escuela como espacio formativo.

Por último, un elemento novedoso para el análisis de cómo mejorar la calidad de la formación en la educación básica, refiere a la modalidad consecutiva para este nivel, presente en todos los países estudiados y ausente en el escenario nacional. Los estudiantes que ingresan a formarse como profesores de educación básica también pueden acceder a una modalidad consecutiva de uno o dos años, siempre que demuestren haber aprobado en sus estudios superiores anteriores, un número determinado de créditos en las áreas principales del currículum (lenguaje, matemática, ciencias sociales y ciencias) $\mathrm{u}$ otras que se estimen pertinentes. Esta alternativa abre un campo de análisis y discusión de gran interés, ya que en nuestro país los resultados de las pruebas Inicia y de TEDs-M muestran debilidades en la formación de la educación básica que no se sabe muy bien cómo remontar.

\subsection{En relación con los procesos de acreditación}

Aunque existen algunos matices en cuanto al grado de control de los estados y al tipo de agencias que realizan la acreditación (ligadas al ministerio de educación o privadas), los países analizados muestran procesos de acreditación relativamente parecidos. Con relación a nuestro país, la gran diferencia es que en los procesos de acreditación los estándares de la profesión docente tienen un lugar importante. Esto quiere decir que los estándares en estos países no están referidos 
a pruebas o exámenes a los estudiantes, sino que se orientan al diseño y ajuste de los programas de formación. Es así que los procesos de acreditación consisten en demostrar que el programa responde a los estándares, lo que puede traducirse en los objetivos, contenidos y actividades de los cursos, en prácticas, seminarios o trabajos de los alumnos. Es la facultad de educación la responsable de definir los equipos y procedimientos de planificación, monitoreo y ajuste internos que permitan alcanzar los estándares nacionales, estaduales o provinciales. Prevalecería en las políticas educativas de los casos estudiados una concepción de la evaluación de la formación inicial de tipo participativa, en la que se confía a las instituciones formadoras el logro de los estándares, mediante procesos internos de mejoramiento continuo antes que por la vía de exámenes, que ponen el acento en el logro individual de los estudiantes al egreso. Para las instituciones a cargo de la formación docente, este mejoramiento permanente de sus programas asegura la calidad de las competencias de sus egresados y no sería necesario organizar pruebas especiales que midan estos conocimientos y habilidades.

En nuestro país, la idea de que los estándares nacionales para egresados de carreras de pedagogía ${ }^{6}$ orienten el diseño de las mallas curriculares de formación, ha estado en la base de los equipos técnicos elaboradores y consultores. Es más, antes de haber sido divulgados como documento oficial del Ministerio de Educación, ya estaban siendo analizados y usados por distintas universidades en sus rediseños curriculares o ajustes de cursos, lo que muestra la necesidad de contar con este tipo de instrumentos para el diseño de modelos formativos de profesores.

Podemos destacar de este estudio el rol que podrían jugar en un futuro próximo los estándares disciplinarios y pedagógicos en los procesos de acreditación de carreras de pedagogía. En la medida en que estos estándares han sido construidos de manera colaborativa y consensuada con las propias universidades, pensamos que estos pueden constituirse en un referente legítimo para orientar

6 Estándares disciplinarios y pedagógicos en las áreas de lenguaje, matemática, ciencias y ciencias sociales para los y las egresadas de educación parvularia, básica y media. 
120 ESTÁNDARES Y REGULACIÓN DE CALIDAD DE LA FORMACIÓN DE PROFESORES: DISCUSIÓN DEL CASO CHILENO DESDE UNA PERSPECTIVA COMPARADA - Carmen Sotomayor, Jacqueline Gysling

y fortalecer los actuales procesos de acreditación. El contar con una base de conocimientos de la profesión docente, expresada en estos estándares, permite pedir a los programas de formación de profesores ser consistentes con los contenidos y habilidades allí propuestos, y a las agencias acreditadoras guiarse por estos acuerdos. Asimismo, la unánime demanda de contar con profesores de calidad en nuestro país, nos hace pensar que es el momento de impedir que las carreras de pedagogía no acreditadas continúen formando profesores. De este modo, los procesos de acreditación podrían tener un impacto efectivo en el mejoramiento de la calidad de los futuros docentes.

\subsection{La certificación para el ejercicio profesional}

La certificación nacional o estadual de los docentes egresados es común en todos los casos estudiados. En Nueva York, Victoria e Inglaterra se otorga una certificación provisoria a los egresados que les permite ejercer por uno o dos años, durante o al cabo de los cuales deben demostrar su capacidad para enseñar en forma definitiva. En el caso de Quebec, este proceso está incluido en la formación, de modo que quienes han realizado sus estudios en las universidades del estado acreditadas, acceden directamente a la certificación definitiva. Tras la política de certificación provisoria está el principio de que el profesor que recién sale de la universidad no puede todavía demostrar de manera suficiente las capacidades necesarias para ejercer la enseñanza. Estas competencias solo podrá lograrlas luego de algunos años de práctica profesional real. La concepción de la formación docente como un aprendizaje permanente y fundado en conocimientos a la vez teóricos y prácticos es muy sólida en estos países, lo que se expresa en los mecanismos de certificación descritos.

Para obtener la certificación, los futuros profesores deben pasar por pruebas de conocimientos, como es el caso de Nueva York; de habilidades básicas en lenguaje, matemática y TICS, en Inglaterra; o de francés en el caso de Quebec. Estas pruebas no guardan relación con los estándares generales definidos a nivel estadual. En todos estos casos, un antecedente fundamental para la certificación es el informe realizado por los profesores mentores y los directores de establecimientos donde ejercen en forma provisional. 
En el proceso de certificación, junto con resguardar que los docentes tengan las competencias requeridas para la profesión, se supervisa que los profesores no tengan antecedentes judiciales, considerando que serán profesionales a cargo de niños. Además, realizar la certificación por un órgano estatal les permite contar con un registro centralizado de docentes. Este registro les da una visión detallada del cuerpo de profesores y, a partir de ello, pueden informar a las universidades sobre requerimientos de profesores e incluso fijar cuotas de vacantes en la formación, como en el caso de Inglaterra y Canadá.

No existe hasta ahora en nuestro país un sistema de certificación o habilitación profesional como el de los casos estudiados. El proyecto de examen de excelencia profesional, anteriormente mencionado, podría apuntar en esta dirección. Sin embargo, es voluntario y se realizaría al egreso de los estudiantes, buscando medir solo conocimientos disciplinarios, un componente importante del desempeño de un profesor, pero en ningún caso el único.

De acuerdo con lo observado en los casos en estudio, para medir las capacidades docentes es indispensable evaluar desempeños o competencias (como preparación de clases, manejo de la disciplina, atención diferenciada a los alumnos, entre otros). Medir competencias supone pensar en otros sistemas de evaluación durante los primeros años de profesión (y no solo al egreso de la formación inicial) e implica también introducir dispositivos de apoyo o mentoría, de modo que los profesores novicios puedan demostrar buenos desempeños y deseen mantenerse en la profesión. Se sabe que la inserción profesional de docentes en las escuelas es un tema crítico y que altos porcentajes de abandono se producen en el curso de los cinco primeros años de ejercicio. La inversión que está realizando el país en atraer a jóvenes talentosos a la pedagogía, podría no tener los resultados esperados sino se toman medidas en este sentido. Lo anterior supondría, además, alinear los estándares de egreso de la formación inicial con los estándares de formación en servicio (nuestro actual Marco para la Buena Enseñanza), estableciendo un marco que oriente el desarrollo profesional docente concebido como un continuo. 
122 ESTÁNDARES Y REGULACIÓN DE CALIDAD DE LA FORMACIÓN DE PROFESORES: DISCUSIÓN DEL CASO CHILENO DESDE UNA PERSPECTIVA COMPARADA - Carmen Sotomayor, Jacqueline Gysling

No se ha discutido en el país la relación entre la certificación y la posibilidad de contar con un registro centralizado de profesores ni tampoco sobre la forma de instalar ciertas barreras éticas orientadas a la protección de los escolares, lo cual sería necesario debatir.

\subsection{Los estándares de formación}

Como se ha señalado antes, los estándares de los países estudiados engloban la generalidad de los ámbitos más importantes de la profesión: enseñanza, conocimiento del contexto y de los estudiantes, y compromiso profesional, siendo algunos más generales y otros más detallados. En Estados Unidos existen también estándares que orientan las condiciones institucionales de la formación inicial (los estándares del NCATE) y, en el caso de Canadá, se observan estándares que propician la interpretación crítica de los saberes y de la cultura, como parte de las competencias docentes. Casi todos los entrevistados de las universidades analizadas manifestaron que los estándares son orientadores de los programas de formación, pero que estas agregan sus propios énfasis, por ejemplo, poner acento en formar personas reflexivas (Nueva York) o comprensivas de la diversidad cultural (Quebec). Prefieren estándares menos detallados y menos prescriptivos que les permitan incorporar su visión propia. El caso de Inglaterra, por ejemplo, revela una apreciación crítica de políticas de mayor control de las últimas décadas y un cuestionamiento, producto de las bajas en los resultados internacionales. No obstante, varios entrevistados al mismo tiempo perciben que el dominio disciplinario no está suficientemente desarrollado y que los estándares resultan muy genéricos en este aspecto. Es así que algunos sugieren una suerte de estándares en distintos "pisos" o niveles, de más generales y englobadores (y también más obligatorios) a más detallados y específicos (más orientadores).

Esta observación es interesante y pertinente para nuestro país, por cuanto el Marco para la Buena Enseñanza, que es el referente de la evaluación docente, puede entenderse como la definición general de estándares profesionales. En tanto, los estándares disciplinarios y pedagógicos que se han elaborado en el contexto del Programa Inicia, pueden considerarse una definición más pormenorizada de 
los conocimientos y habilidades que deberían desarrollarse durante el proceso de formación. Desde nuestro punto de vista, ambas herramientas debieran ser parte de una misma construcción que oriente tanto la formación inicial, como a los profesores en servicio, dando mayor consistencia al proceso de desarrollo profesional de los docentes.

\subsection{Perspectivas}

Como se ha descrito antes, es interpelante el cuidado y exigencia que en los estados estudiados se pone a la formación de profesores y su habilitación para ejercer en forma definitiva la profesión. En nuestro país se están diseñando estándares de formación de profesores y se están adoptando un conjunto de medidas que buscan mejorar su calidad, sin embargo, a partir de los casos analizados nos preguntamos si lo que se está definiendo será suficiente. Al analizar las medidas propuestas desde una perspectiva sistémica, nos parece que falta una reflexión más global sobre la política de formación de profesores que permita asegurar que se están abordando las dimensiones centrales del problema.

Un paso positivo es definir estándares de formación de profesores; ello ha significado abordar sistemáticamente la pregunta por el conocimiento base de la profesión docente. Esta elaboración, que ha sido amplia y participativa, tiene un valor en sí misma, ya que se establece un referente curricular para el diseño de los programas de formación, del cual se carecía. Sin embargo, tener estándares no es sinónimo de tener una política de formación de profesores basada en estándares. En esta línea, nos parece que lo que se está desarrollando en Chile es todavía inicial.

A la luz de los casos analizados, se destaca la relevancia de los procesos de acreditación para resguardar la calidad de los procesos formativos. En nuestro país, la acreditación de carreras de pedagogía es obligatoria, pero solo para recibir recursos del Estado, lo que significa que las instituciones que no sean acreditadas, pueden seguir preparando profesores. Además, no se prevé en los proyectos legislativos en curso que la acreditación sea conducida por una agencia 
124 ESTÁNDARES Y REGULACIÓN DE CALIDAD DE LA FORMACIÓN DE PROFESORES: DISCUSIÓN DEL CASO CHILENO DESDE UNA PERSPECTIVA COMPARADA - Carmen Sotomayor, Jacqueline Gysling

especializada en formación de profesores, como ocurre en los países estudiados, ni que se realice sobre la base de los estándares que se están definiendo.

Otro aspecto que llama la atención es la forma adoptada en nuestro país por la prueba Inicia, que se caracteriza por evaluar a los egresados de pedagogía en contenidos, principalmente disciplinarios, lo que no tiene precedente en ninguno de los casos analizados. En estos países se evalúa el desempeño en los primeros años de ejercicio profesional para certificar a los nuevos docentes. El único caso donde se aplican pruebas de conocimiento es el estado de Nueva York. Sin embargo, este estado invierte en formación continua para que los postulantes superen con éxito las pruebas, liberando a las instituciones formadoras de esta responsabilidad. Al mismo tiempo, Nueva York está introduciendo modificaciones para incorporar a partir de 2013 una evaluación de desempeño.

La prueba Inicia, como sistema que provee información a las universidades para revisar sus procesos de formación y a los postulantes para la selección de carreras, puede ser una innovación interesante, sin embargo, preocupa que su énfasis en los conocimientos disciplinarios pueda debilitar los vínculos de la formación con la práctica que, de acuerdo a los casos estudiados, habría que fortalecer.

Por último, nos parece que podría considerarse la existencia de una institucionalidad especializada, ya sea una agencia o una dependencia del Ministerio de Educación responsable de los estándares y de la política de formación de profesores. Esta institucionalidad podría tener entre sus funciones favorecer la implementación de estándares, completar la formulación de estándares para todas las áreas del currículum y avanzar hacia el establecimiento de un registro de profesores que ingresan al sistema.

Aunque el país avanza con medidas que se orientan a mejorar la calidad de la formación de profesores, el estudio realizado nos muestra que estamos distantes de tener una política de formación de profesores basada en estándares, que considere de manera articulada dispositivos como la obligatoriedad efectiva de la acreditación de 
carreras, certificación para ejercer la profesión y registro de los nuevos profesores. Si bien lo que se está diseñando en Chile se asemeja en algunos aspectos a los casos analizados, el sistema de formación docente se mantiene fundamentalmente desregulado.

La importancia que reviste la formación de profesores amerita continuar realizando investigación y seguimiento del proceso que se ha iniciado en nuestro país. En la perspectiva de futuras investigaciones comparadas, parece especialmente relevante analizar lo que está ocurriendo en el contexto latinoamericano, particularmente en países cercanos a Chile que han seguido un camino distinto. Es el caso de Argentina, Uruguay y Perú que han definido en sus leyes generales de educación que la formación docente sea regulada por el Estado, y han establecido la creación de una institucionalidad centralizada única para definir las políticas y lineamientos curriculares de la formación inicial de los docentes.

Este estudio contó con la colaboración de Valentina Abufhele, Alejandra Arratia, Elisa de Padua, Rocío Esquivel, a quienes agradecemos su participación.

\section{Referencias bibliográficas}

Ávalos, B. y Matus, C. (2010) La formación inicial docente en Chile desde una óptica internacional. Informe nacional del Estudio Internacional IEA TEDS-M. Santiago: Ministerio de Educación.

Baker, E. (2004) Aligning Curriculum, Standards, and Assessments: Fulfilling the Promise of School Reform. CSE Report 645, CRESST, University of California, Los Ángeles, USA.

Banco Mundial (2007) El diseño institucional de un sistema efectivo de aseguramiento de la calidad de la educación en Chile. Documento Banco Mundial.

Bolívar, A. (2003) Si quiere mejorar las escuelas, preocúpese por capacitarlas. El papel del rendimiento de cuentas por estándares en la mejora. Profesorado, revista de curriculum y formación del profesorado, 7 (1-2), pp. 1-11.

Belleï, C. y Valenzuela, J.P. (2010) ¿Están las condiciones para que la docencia sea una profesión de alto estatus en Chile? En S. Martinic y G. Elacqua 
126 ESTÁNDARES Y REGULACIÓN DE CALIDAD DE LA FORMACIÓN DE PROFESORES: DISCUSIÓN DEL CASO CHILENO DESDE UNA PERSPECTIVA COMPARADA - Carmen Sotomayor, Jacqueline Gysling

(editores). ¿Fin de ciclo? Cambios en la gobernanza del sistema educativo. Santiago: UNESCO-OREALC/ Pontificia Universidad Católica de Chile.

CEPPE (2011) Notas para Educación, enero, 2011.

Comisión Asesora Presidencial para la Calidad de la Educación (2006) Informe Final. Santiago: Gobierno de Chile.

Darling-Hammond, L. (2001) El derecho de aprender. Crear buenas escuelas para todos. Barcelona: Ariel.

Darling-Hammond, L. (2007) Evaluating 'No Child Left behind'. The Nation, mayo 21 .

Darling-Hammond, L. (2010) Teacher Education and the American Future. Journal of Teacher Education 61 (1-2), pp. 35-47.

Elliott, J. (2002) La reforma educativa en el Estado evaluador. Perspectivas. Vol. XXXII, n. ${ }^{\circ}$ 3, septiembre 2002. Recuperado julio 2011 en: http://firgoa. usc.es/drupal/node/40076 ().

Elmore, R. (2003) Salvar la brecha entre estándares y resultados: el imperativo para el desarrollo profesional en educación. Profesorado, revista de currículum y formación del profesorado. N. ${ }^{\circ}$ 7, pp. 9-39.

Ferrer, G. (2006) Estándares en educación. Implicancias para su aplicación en América Latina. PREAL.

Forster, M. (2002) Estándares de Aprendizaje. Unidad de Currículo y Evaluación. Transcripción de la presentación de Margaret Forster en el edificio telefónica. MINEDUC.

Hansche, L.; Hambleton, R.; Mills, C.; Jaeger, R. y Redfield, D. (1998) Handbook for the Development of performance Standards: Meeting the Requirements of Title I. Preparado por U.S. Department of Education and The Council of a Chief State School Officers. Maryland: Frost Associates.

Linn, R. y Herman, J. (2001) La evaluación impulsada por estándares: problemas técnicos y políticos en la medición del progreso de la escuela y los estudiantes. Documento del Programa de Promoción de la Reforma Educativa en América Latina y el Caribe (PREAL) - GRADE.

Ministerio de Educación (2001) Estándares de desempeño para la formación inicial de docentes. Recuperado en junio de 2011 de: http://www. educarchile.cl/Userfiles/P0001/File/estandares_formacion_docentes. pdf

Ministerio de Educación, CPEIP (2008) Marco para la Buena Enseñanza. Recuperado en junio de 2011 de: http://www.docentemas.cl/docs/ MBE2008.pdf 
Ministerio de Educación (2010) Evaluación diagnóstica Inicia. Resultados aplicación 2009. Santiago: CPEIP.

National Research Council (2010) Preparing teachers: Building evidence for sound policy. Committee on the Study of Teacher Preparation Programs in the United States, Center for Education. Division of Behavioral and Social Sciences and Education. Washington, DC: The National Academies Press.

Panel de expertos para una educación de calidad (2010) Informe final: primera etapa. Propuestas para fortalecer la profesión docente en el sistema escolar chileno. Informe entregado al Ministro de Educación en julio de 2010.

PISA OECD (2004) What Makes School Systems Perform? Seeing School Systems Through the Prism of PISA.

Ravitch, D. (1996) Estándares nacionales en educación. Documento PREAL, diciembre 1996.

Vlasceanu, L.; Grünberg, L. y Parlea, D. (comps.) (2007) Quality Assurance and Accreditation: A Glossary of Basic Terms and Definitions. Bucarest: UNESCO-CEPES.

Zuzovsky, R. y Libman, Z. (2006) Standards of Teaching and Teaching Test: Is the Right Way to Go? Studies in Educational Evaluation 32. pp. 37-52.

\section{Documentos oficiales de los países estudiados}

Australia

Australian Institute for Teaching and School Leadership (AITSL) (2010) National Professional Standards for Teachers. Draft. En: http://www. aitsl.edu.au/ta/go

Australian Institute for Teaching and School Leadership (AITSL) (2011) National Professional Standards for Teachers.

Victoria Institute of Teaching (2011) Aplication for Registration. Form A.

Victoria Institute of Teaching (2011) Standards of Professional Practice for Full Registration.

\section{Canadá}

Comité d'agrément des programmes de formation (2011) Lagrément des programmes de formation à l'enseignement: un processus cohérent et équitable. Sitio internet www.capfe.gouv.qc.ca.

Gobierno de Quebec. (2001) La formation à l'enseignement: les orientations et les compétences professionnelles. Quebec: Ministère de l'Éducation. 
128 ESTÁNDARES Y REGULACIÓN DE CALIDAD DE LA FORMACIÓN DE PROFESORES: DISCUSIÓN DEL CASO CHILENO DESDE UNA PERSPECTIVA COMPARADA - Carmen Sotomayor, Jacqueline Gysling

Gobierno de Quebec, Ministerio de la educación. (2011) L'Éducation au Quebec en bref. Consultado el 4 de mayo 2011 en: http://www.mels.gouv.qc.ca/ scolaire/educqc/pdf/educqcfra.pdf.

Sitio del Comité d'agrément des programmes de formation à l'enseignement (CAPFE): http://www.capfe.gouv.qc.ca/

Estados Unidos

Council of Chief State School Officers (2010) Model Core Teaching Standards: A Resource for State Dialogue (Draft for Public Comment). Interstate Teacher Assessment and Support Consortium (InTASC) Washington, DC: Author.

Interstate New Teacher Assessment and Support Consortium (1992) Model Standards for Beginning Teacher Licensing, Assessment and Development: A Resource for State Dialogue. Washington DC.

National Board for Professional Teaching Standards (NBPTS) (2002) What Teachers Should Know and Be Able to Do. Disponible en: http://www. nbpts.org/UserFiles/File/what_teachers.pdf

National Council for Accreditation of Teacher Education (NCATE) (2008) Professional Standards for the Accreditation of Teacher Preparation Institutions. Washington DC.

National Council for Accreditation of Teacher Education (NCATE) (2010) Transforming Teacher Education Through Clinical Practice. A National Strategy to Prepare Effective Teachers. Report of the Blue Ribbon Panel on clinical preparation and partnerships for improved student learning. Washington DC.

New York State Education Department (NYSED) (2011) The New York State Teaching Standards. Enero 11, 2011. Recuperado en mayo 2011 en: http://www.highered.nysed.gov/tcert/resteachers/teachingstandardsl. html

Sitio de New York State Teacher Certifications Examinations: www.nystce. nesinc.com

Inglaterra

Department for Education DfE (2010) The Importance of Teaching. White Paper Equalities Impact Assessment. Recuperado en junio 2011 en: https:// www.education.gov.uk/publications/eOrderingDownload/CM-7980Impact_equalities.pdf

Department for Education (DfE) (2011) Teachers' Standards. Efective from 1 September 2012. Recuperado en julio de 2011 en: http://media. education.gov.uk/assets/files/pdf/t/teachers standards.pdf 
Ofsted (2008) Framework for the Inspection of Initial Teacher Education 200811. www.ofsted.gov.uk

Training and Development Agency for Schools (TDA) (2008) Professional Standards for Teachers. Why Sit Still in Your Career? (Documento oficial).

Sitio del General Teaching Council: http://www.gtce.org.uk/ 